\title{
Reliability analysis of a large-scale landslide using SOED-based RSM
}

\author{
Xiao-Cheng Huang ${ }^{1,2} \cdot$ Xiao-Ping Zhou ${ }^{1,2,3}$
}

Received: 31 July 2017 / Accepted: 13 November 2017 / Published online: 27 November 2017

(c) The Author(s) 2017. This article is an open access publication

\begin{abstract}
A design matrix in response surface method (RSM) that satisfies the orthogonality is very useful because the mean square error can be minimized, so that the response surface is more precise. But the orthogonality of a second-order design matrix in conventional RSM cannot be satisfied. In this paper, a second-order orthogonal experimental design (SOED)-based RSM is proposed by considering the orthogonality of high-order design matrix. The SOED is constructed by changing the length of star points, and the main characteristic of SOED is that the design matrix is diagonal. When the high-order terms are considered in the SOED-based RSM, a globe optimal solution can be found. As the regression equation is determined, the reliability index can be analyzed by the normalized distance between the mean value of the performance function and the critical limit state of the safety factor. A practical large-scale landslide with two slip surfaces is taken to verify the applicability and precision of the proposed method in detail. It is found that the SOED-based response surface is more rigorous than the conventional RSM.
\end{abstract}

Keywords Landslides $\cdot$ Response surface method (RSM) · Second-order orthogonal experimental design (SOED) $\cdot$ Monte Carlo simulations $\cdot$ Reliability analysis

\section{Introduction}

Landslide or slope failure may happen even it is designed with high safety factors because geomaterials are mostly dominated by uncertainties (He et al. 2010; Johari and Lari 2016). Hence, the performance of landslides determined by a factor of safety cannot characterize uncertainties explicitly and sufficiently (Pourghasemi et al. 2014; Wang et al. 2016) and may sometimes be inclined to yield misleading results (Duncan and Buchignani 1973). Fortunately, reliability analyses, such as the first-order second-moment method (FOSM) (Low 2013), Monte Carlo simulations (MC) (Wang et al. 2010; Wang 2012) and response surface methodology(RSM)

Xiao-Ping Zhou

xiao_ping_zhou@126.com

1 School of Civil Engineering, Chongqing University, Chongqing 400045, People's Republic of China

2 State Key Laboratory of Coal Mine Disaster Dynamics and Control, Chongqing University, Chongqing 400044, People's Republic of China

3 Key Laboratory of New Technology for Construction of Cities in Mountain Area, Chongqing 400045, People's Republic of China
(Li et al. 2015), were applied to quantify these uncertainties in recent years (Phoon and Kulhawy 1999; Phoon 2008; Li et al. 2009, 2011; Ching and Phoon 2012; Zhang et al.2012), which emerge as a more reasonable and rigorous way to handle uncertainties (Ji et al. 2012; Tang et al. 2013; Hicks et al. 2014).

RSM is used to determine how a response is affected by a set of quantitative factors over some specified region. This information can be used to optimize the settings of a process to give a maximum or minimum response. For a given number of variables, response surface analysis techniques require more trials than the two-level fractional factorial design techniques. In order to calculate simply, most cases do not include cross-terms and second-order terms in response function. Although this greatly simplifies the number of sampling points, it may not be appropriate in all cases (Box and Draper 2006). The results obtained from a first-order response surface may be local optimal solutions (Myers and Raymond 2012). Hence, it is necessary to find a globe optimal solution by using a high-order response surface.

In the conventional RSM, the first-order response surface cannot fit well the curvature of a true underlying surface, solutions may be wrong and the selection of the grid 
of experimental design points has no precise guidelines or theory in RSM (Guan and Melchers 2001). In SOED, the cross-correlations of uncertain variables are assumed to be 0 , and the orthogonality of design matrix containing highorder terms is satisfied by changing the length of star points so that the variance of the predicated values and target values is minimized. In order to overcome the shortcomings of the conventional RSM, RSM is combined with SOED, which is called the SOED-based RSM. In the SOED-based RSM, not only the response surface is more precise, but also the significance of regression coefficients is easy to test because the sum of error between the fitting equation and the regression equation is equal to 0 . Moreover, the orthogonality of the first-order terms, cross-terms and quadratic terms in design matrix is satisfied. The design matrix obtained by the proposed method can contribute to the selection of sampling points. Example of reliability analysis of a large-scale landslide is taken to prove the validity and capability of the proposed method. Firstly, the values of uncertain variables, such as the friction angle and cohesion, are transformed from encoded variables in SOED design matrix. Then, the response value, the factor of safety $(F S)$, is calculated by $3 \mathrm{D}$ rigorous limit equilibrium method. Finally, the reliability index can be obtained by MATLAB optimization toolbox.

\section{Construction of second-order orthogonal experimental design (SOED)}

SOED is based on orthogonal Latin squares and group theory (Hall 1959; Johnson and Johnson 1991). The main characteristic of SOED is that the design matrix is diagonal; meanwhile, the sum of error between the theoretical response values by fitting equation and the actual response values is equal to 0 .

\section{Sampling points}

The sampling points in an orthogonal experimental design are consisted of corner points, star points and mean points. The corner points are located at the corner of distribution of experimental points, the star points are located in the axis and the mean points are located at origin, as shown in Figs. 1 and 2. The length from the star points to the mean points is $\gamma$, which is introduced in the next section. It can be obviously found from Figs. 1 and 2 that the run by mean points is 1 , the run by star points is $2^{m}$ ( $m$ is the total number of variables) and the run by corner points is $2^{m}$. Hence, the total number of SOED experimental times is:

$N=2^{m}+2 m+1$

It should be pointed out that the corner points can be taken by factorial design $\left(2^{k}\right.$ design $)$, which requires a large

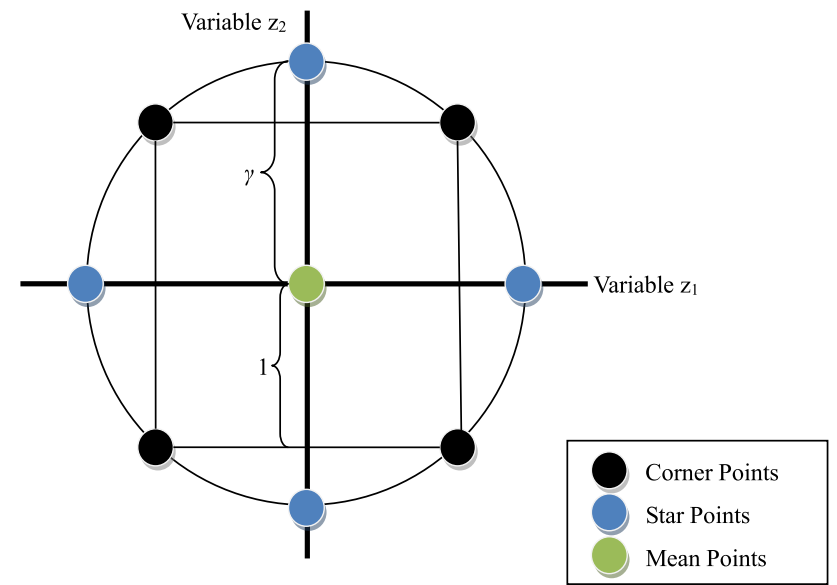

Fig. 1 Distribution of design points in SOED with two variables

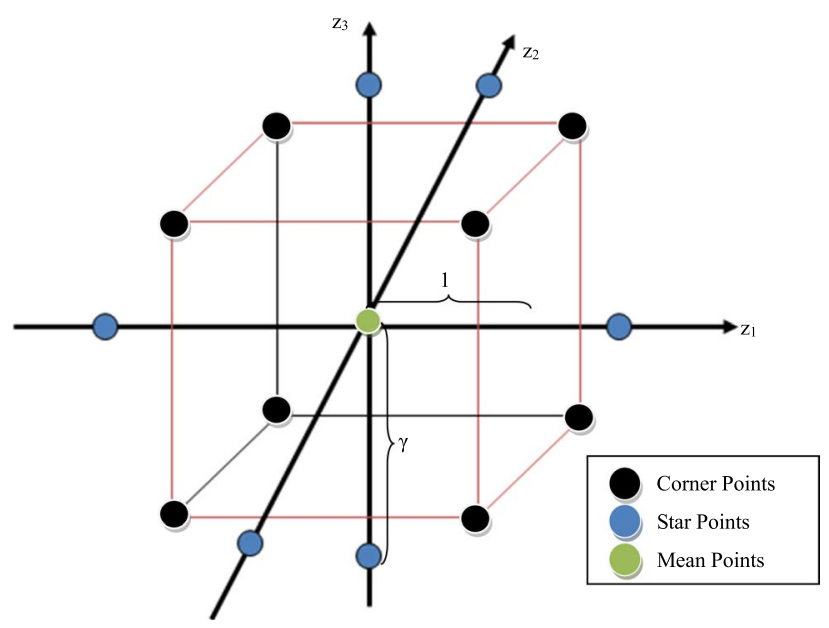

Fig. 2 Distribution of design points of the SOED with three variables

number of design points, as listed in Table 1 (four variables). In general, a $2^{-p}$ th fraction of a $2^{k}$ design consists of $2^{k-p}$ points from a full $2^{k}$ design $(p<k)$. It is assumed that the number of runs is $m_{\mathrm{c}}$, for a whole fraction design, $m_{\mathrm{c}}$ is $2^{m}$, a one-half fraction design $m_{\mathrm{c}}$ is $2^{m-1}$ and a one-fourth fraction design $m_{\mathrm{c}}$ is $2^{m-2}$.

It can be found from Table 1 that $\sum_{j=1}^{N} z_{j}=0$ and $\sum_{i \neq j} z_{i} z_{j}=0$, i.e., the matrix is an orthogonal one. However, the orthogonality of the matrix cannot be satisfied when the second-order terms are considered. For instance, $\sum_{i \neq j} z_{i}^{2} z_{j} \neq 0$, or $\sum_{i \neq j} z_{i} z_{j}^{2} \neq 0$.

\section{The length of star points}

In order to construct a second-order orthogonal matrix, the length of star points $\gamma$ should be carefully taken. Firstly, the quadratic terms should be centralized, and it can be written in the following form: 
Table 1 Encoded design variables (or matrix) by factorial design with four variables

\begin{tabular}{rrrr}
\hline $\mathrm{z}_{1}$ & $\mathrm{z}_{2}$ & $\mathrm{z}_{3}$ & \multicolumn{1}{c}{$\mathrm{z}_{4}$} \\
\hline 1 & 1 & 1 & 1 \\
1 & 1 & 1 & -1 \\
1 & 1 & -1 & 1 \\
1 & 1 & -1 & -1 \\
1 & -1 & 1 & 1 \\
1 & -1 & 1 & -1 \\
1 & -1 & -1 & 1 \\
1 & -1 & -1 & -1 \\
-1 & 1 & 1 & 1 \\
-1 & 1 & 1 & -1 \\
-1 & 1 & -1 & 1 \\
-1 & 1 & -1 & -1 \\
-1 & -1 & 1 & 1 \\
-1 & -1 & 1 & -1 \\
-1 & -1 & -1 & 1 \\
-1 & -1 & -1 & -1 \\
\hline
\end{tabular}

$z_{i j}^{\prime}=z_{i j}^{2}-\frac{1}{N} \sum_{i=1}^{N} z_{i j}^{2}=z_{i j}^{2}-\left(m_{c}+2 \gamma^{2}\right) / N$

\section{SOED-based RSM}

A second-order RSM can be written as:

$\hat{y}=\alpha+\sum_{i=1}^{m} \beta_{i} z_{i}+\sum_{j<i} \beta_{i j} z_{i} z_{j}+\sum_{i=1}^{m} \beta_{i i} z_{i}^{2}+\varepsilon, \quad j=1,2, \ldots n-1(j \neq i)$ where $z_{i j}^{\prime}$ is the centralized quadratic terms, $z_{i j}$ is the firstorder terms and $N$ is the total run of experimental design. Since any two second-order terms should satisfy the orthogonality, the following expression can be obtained:
$\sum_{i=1}^{N} z_{i}^{\prime} z_{j}^{\prime}=0$

Substituting (2) into (3), we have

$m_{c}-\left(m_{c}+2 \gamma^{2}\right)^{2} / N=-\frac{4}{N}\left[\gamma^{4}+m_{c} \gamma^{2}-\frac{1}{2} m_{c}\left(m+\frac{1}{2} m_{0}\right)\right]=0$

where $m_{0}$ is the run by the mean points, and it is equal to 1 in most case. Simplifying Eq. (4), the length of star points $\gamma$ in SOED can be obtained as:

$\gamma=\sqrt{\frac{\sqrt{\left(m_{c}+2 m+m_{0}\right) m_{c}}-m_{c}}{2}}$

Consider $m=4$ and the one-half fraction of a $2^{k}$ design is chosen, then $m_{\mathrm{c}}$ is $2^{4-1}=8$. From Eq. (1), $N$ is 17 . From (5), $\gamma$ is 1.353 . The design matrix with four variables is listed in Table 2. 
where $\alpha$ is the constant terms, $\left\{\beta_{i}\right\},\left\{\beta_{i j}\right\}$ and $\left\{\beta_{i i}\right\}$ are, respectively, the regression coefficient of first-order terms, crossterms and second-order terms, $\varepsilon$ is the error, $z_{i}$ and $z_{j}$ are the uncertain variables.

It can be found from Eq. (6) that there is $(m+1)(m+2) / 2$ unknowns. Therefore, the total number of experiments should be larger than $(m+1)(m+2) / 2$.

In the conventional RSM, the response surface is approximated using a conventional regression technique. Therefore, the selection of appropriate sampling points is important. Unfortunately, the selection of the grid of experimental design points has no precise guidelines or theory (Guan and Melchers 2001). In order to calculate simply, Eq. (6), which does not include cross-terms and quadratic terms, is always used as the approximation. This greatly simplifies the number of sampling points, but it may not be appropriate in all cases. However, the high order is the true one, which was also mentioned by Myers and Raymond (2012). Fortunately, the proposed SOED-based RSM takes the second-order terms into consideration, and it can also provide a guideline for sampling strategy in RSM.

The procedure of SOED is the same as the conventional RSM; the sampling points (input variables) should firstly be selected, e.g., the stability of landslide is influenced by the parameters, such as friction angle and cohesion. Then, the values of input variables should be determined. Next, the experiment table is selected, from which the response variables are obtained. Finally, a polynomial function can be determined.

\section{Reliability index $\beta_{\mathrm{RI}}$ for SOED}

The reliability index is the shortest distance from the limit state function to the origin of the transformed space of random variables. It can be defined by (US Army Corps of Engineers 1995)

$$
\left.\begin{array}{l}
\min \beta_{\mathrm{RI}}=\|\mathbf{Y}\|=\sqrt{\mathbf{Y}^{\mathbf{T}} \mathbf{Y}}=\sqrt{\sum_{j=1}^{n}\left[\left(x_{j}-\mu_{j}\right) / \sigma_{j}\right]^{2}}, \\
\text { s.t. }\left\{\begin{array}{l}
G^{\prime}(x)=0 \\
x_{\min } \leq x_{j} \leq x_{\max }
\end{array} j=1,2, \ldots n\right.
\end{array}\right\}
$$

where $x_{j}$ is the random variable, $\mu$ and $\sigma$ are, respectively, the vector of mean values and standard deviation of random variables, $\mathbf{Y}$ is a vector of normalized (transformed) variables and $G^{\prime}(x)$ is limit state function.

Solving the minimum $\beta_{\mathrm{RI}}$ ellipse, which is tangent to the limit state surface, can be iteratively performed using optimization tools, such as Excel Solver or MATLAB optimization toolbox. Once the reliability index $\beta_{\mathrm{RI}}$ is determined,
Table 3 Target reliability indices (US Army Corps of Engineers 1995)

\begin{tabular}{lll}
\hline Probability of failure $P_{f}(\%)$ & Reliability index $\beta$ & $\begin{array}{l}\text { Expected } \\
\text { performance } \\
\text { level }\end{array}$ \\
\hline 0.16 & 1 & Hazardous \\
0.07 & 1.5 & Unsatisfactory \\
0.023 & 2 & Poor \\
0.006 & 2.5 & Below \\
0.001 & 3 & Above \\
0.00003 & 4 & Good \\
0.0000003 & 5 & High \\
\hline
\end{tabular}

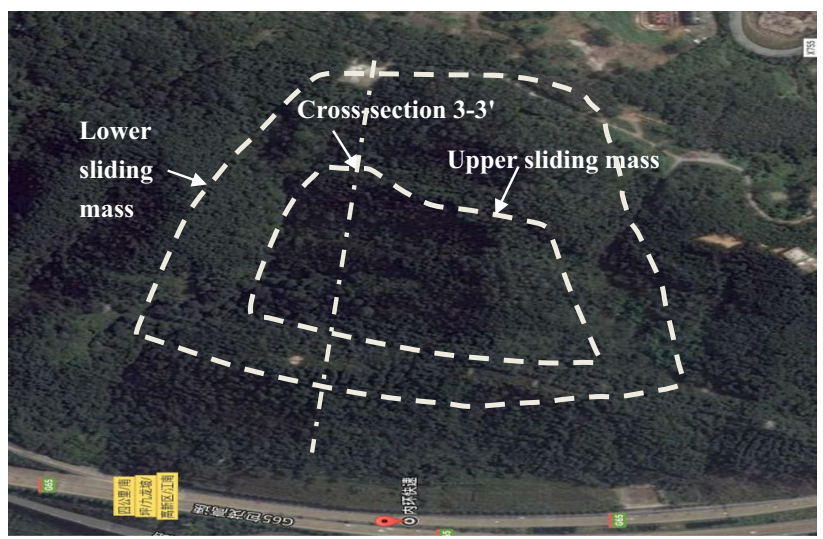

Fig. 3 Photograph of the reinforced Xiangjiashan landslide

the probability of failure can be found. The reliability index $\beta_{\mathrm{RI}}$, the corresponding probability of failure $P_{f}$ and an auxiliary terminology regarding expected performance points are listed in Table 3.

\section{Case study: a large-scale landslide}

In order to demonstrate the efficiency and accuracy of the proposed method, a practical landslide, Xiangjiashan landslide, is taken as an example, and the arbitrary slip surface for this landslide is shown in Fig. 3.

Xiangjiashan landside is located at Chongqing in China. It is observed from Fig. 3 that bird's-eye view of the landslide is an irregular horseshoe shape with the width of $200 \sim 360 \mathrm{~m}$ and longitudinal length of $230 \mathrm{~m}$. The slope angle of the landslide is about $70^{\circ} \sim 80^{\circ}$. The area of the sliding region is approximately $70,000 \mathrm{~m}^{2}$, and the volume of the sliding body is estimated to be $1,400,000 \mathrm{~m}^{3}$. Therefore, Xiangjiashan landslide is a large-scale one (Zhou and Cheng 2015). The landslide was reinforced with the high safety factor during excavation since 1998, but great displacements occurred on June 1, 2004. Therefore, it is indicated that a 
landslide reinforced with high $F S$ cannot be considered as 'safe' one. To study this landslide, the probabilistic way is more reasonable.

According to the geological exploration of Xiangjiashan landslide, there exist two sliding bodies (upper sliding body and lower sliding body), which are observed in Figs. 3 and 4. The upper sliding body contains sandstone, weathered mudstone, and so on. The lower sliding body mainly contains sandstone and weathered mudstone (Zhou and Cheng 2015). It is assumed that unit weight, cohesion and friction angle of geomaterials are in normal distributions, and they are assumed to be uncorrelated. The mechanical parameters are listed in Table 4.

\section{The upper sliding mass}

There are three uncertain variables in this study, i.e., from Eq. (1), $N$ is 15 when $m$ is 3 . From Eq. (5), the length of star points $\gamma$ is 1.215 . In Table 5, the uncertain variables are changed from encoded variables in SOED. For instance, ' 0 ' listed in the column $z_{1}$ is corresponding to mean value $\mu$ ('24.92') of uncertain variable $\eta$, ' \pm 1 ' represents ' $\mu \pm \sigma$, ' \pm 1.215 ' represents ' $\mu \pm 1.215 \sigma$ ', etc.

Sets of response value, $F S$, are obtained by uncertain variables in Table 5 using 3D rigorous limit equilibrium method. $3 \mathrm{D}$ rigorous limit equilibrium method is a more reasonable and rigorous way to calculate $F S$ because six equilibrium equations are satisfied, while parts of equilibrium equations are satisfied in simplified Bishop method and Janbu method. For simplicity, 3D rigorous limit equilibrium method is illustrated in "Appendix."

From Eq. (2), we have $z_{1}^{\prime}=\left(\frac{\eta-24.92}{2}\right)^{2}-0.73$, $z_{2}^{\prime}=\left(\frac{c-55}{5}\right)^{2}-0.73, z_{3}^{\prime}=\left(\frac{\varphi-26}{2}\right)^{2}-0.73$.

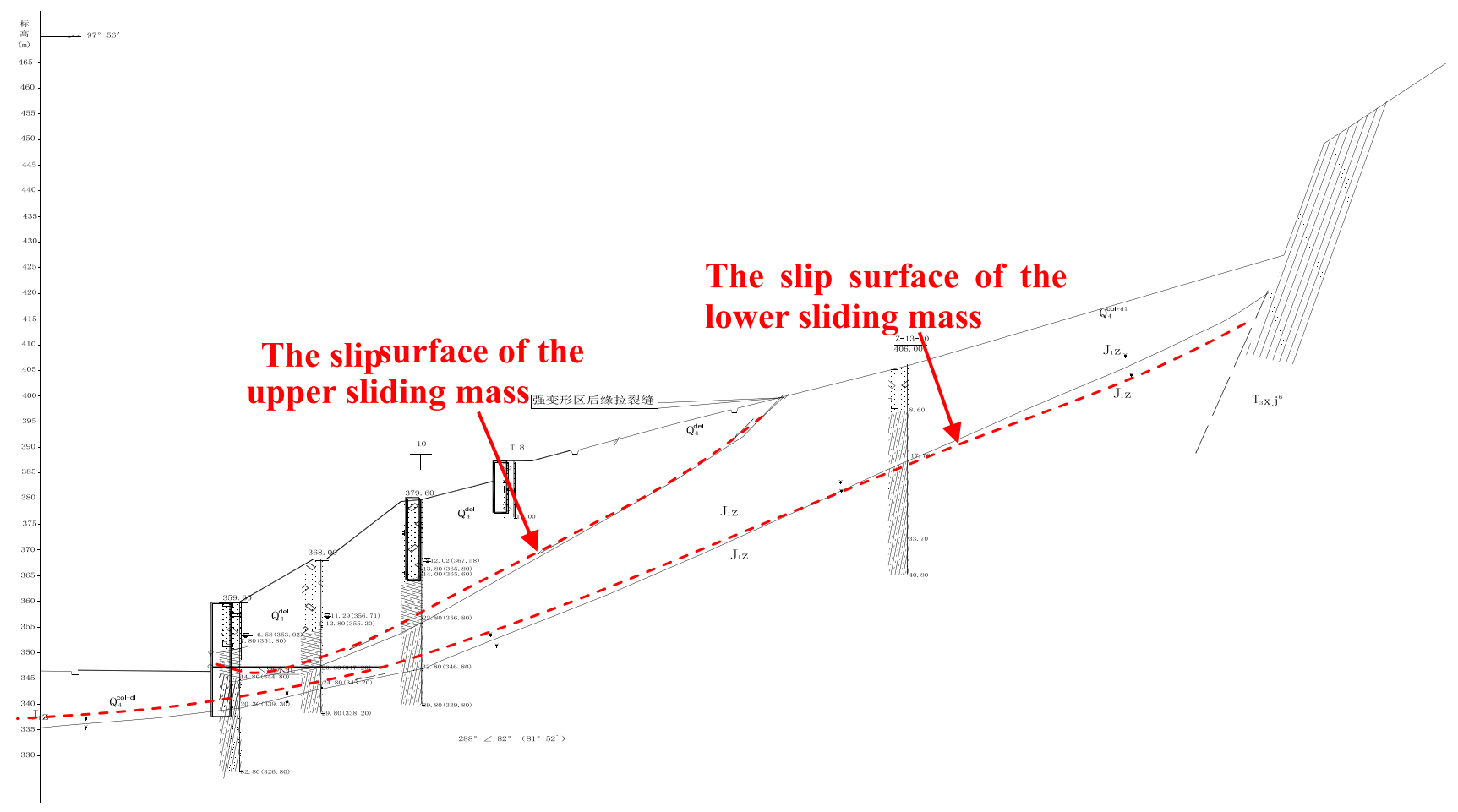

Fig. 4 The 3-3' geological cross section of Xiangjiashan landslides (Zhou and Cheng 2015)

Table 4 Mechanical parameters of the Xiangjiashan landslide

\begin{tabular}{|c|c|c|c|c|c|c|}
\hline \multirow[t]{2}{*}{ Masses } & \multicolumn{2}{|c|}{ Unit weight $x_{1}\left(\eta / \mathrm{kN} \mathrm{m}^{-3}\right)$} & \multicolumn{2}{|c|}{$\begin{array}{l}\text { Cohesion of geomaterials } \\
x_{2}(c / \mathrm{KPa})\end{array}$} & \multicolumn{2}{|c|}{$\begin{array}{l}\text { Internal friction angle of } \\
\text { geomaterials } x_{3}\left(\varphi /{ }^{\circ}\right)\end{array}$} \\
\hline & Mean values & $\begin{array}{l}\text { Standard } \\
\text { deviation }\end{array}$ & Mean values & $\begin{array}{l}\text { Standard } \\
\text { deviation }\end{array}$ & Mean values & $\begin{array}{l}\text { Standard } \\
\text { deviation }\end{array}$ \\
\hline The upper sliding mass & 24.92 & 2 & 55.0 & 5 & 26.0 & 2 \\
\hline The lower sliding mass & 24.92 & 2 & 60.0 & 5 & 25.0 & 2 \\
\hline
\end{tabular}


Table 5 Design matrix in SOED with three variables $(m=3)$

\begin{tabular}{lccrrrrrrrrrrrr}
\hline $\begin{array}{l}\text { Experi- } \\
\text { mental } \\
\text { order }\end{array}$ & $z_{1}$ & $z_{2}$ & $z_{3}$ & $z_{1} z_{2}$ & $z_{1} z_{3}$ & $z_{2} z_{3}$ & $z_{1}{ }^{\prime}$ & $z_{2}{ }^{\prime}$ & $z_{3}{ }^{\prime}$ & $\eta$ & $c$ & $\varphi$ & Response $F S$ \\
\hline 1 & 1 & 1 & 1 & 1 & 1 & 1 & 0.27 & 0.27 & 0.27 & 26.92 & 50 & 28 & 0.996 \\
2 & 1 & 1 & -1 & 1 & -1 & -1 & 0.27 & 0.27 & 0.27 & 26.92 & 50 & 24 & 1.146 & \\
3 & 1 & -1 & 1 & -1 & 1 & -1 & 0.27 & 0.27 & 0.27 & 26.92 & 60 & 28 & 1.191 \\
4 & 1 & -1 & -1 & -1 & -1 & 1 & 0.27 & 0.27 & 0.27 & 26.92 & 60 & 24 & 1.041 \\
5 & -1 & 1 & 1 & -1 & -1 & 1 & 0.27 & 0.27 & 0.27 & 22.92 & 50 & 28 & 1.185 \\
6 & -1 & 1 & -1 & -1 & 1 & -1 & 0.27 & 0.27 & 0.27 & 22.92 & 50 & 24 & 1.035 \\
7 & -1 & -1 & 1 & 1 & -1 & -1 & 0.27 & 0.27 & 0.27 & 22.92 & 60 & 28 & 1.238 \\
8 & -1 & -1 & -1 & 1 & 1 & 1 & 0.27 & 0.27 & 0.27 & 22.92 & 60 & 24 & 1.088 \\
9 & 1.215 & 0 & 0 & 0 & 0 & 0 & 0.747 & -0.73 & -0.73 & 27.35 & 55 & 26 & 1.088 \\
10 & -1.215 & 0 & 0 & 0 & 0 & 0 & 0.747 & -0.73 & -0.73 & 22.49 & 55 & 26 & 1.141 \\
11 & 0 & 1.215 & 0 & 0 & 0 & 0 & -0.73 & 0.747 & -0.73 & 24.92 & 61.07 & 26 & 1.142 \\
12 & 0 & -1.215 & 0 & 0 & 0 & 0 & -0.73 & 0.747 & -0.73 & 24.92 & 48.93 & 26 & 1.083 \\
13 & 0 & 0 & 1.215 & 0 & 0 & 0 & -0.73 & -0.73 & 0.747 & 24.92 & 55 & 28.43 & 1.205 \\
14 & 0 & 0 & -1.215 & 0 & 0 & 0 & -0.73 & -0.73 & 0.747 & 24.92 & 55 & 23.57 & 1.023 \\
15 & 0 & 0 & 0 & 0 & 0 & 0 & -0.73 & -0.73 & -0.73 & 24.92 & 55 & 26 & 1.113 \\
\hline
\end{tabular}

From Eq. (6), the second-order regression equation can be obtained as follows:

$$
\begin{aligned}
\hat{y}_{\text {up - soed }}= & 1.1143-0.0216 z_{1}-0.0114 z_{2}+0.0476 z_{3} \\
& +0.002 z_{1} z_{2}-0.0375 z_{1} z_{3}-0.0375 z_{2} z_{3} \\
& +0.0024 z_{1}^{\prime}+0.001 z_{2}^{\prime}+0.002 z_{3}^{\prime}
\end{aligned}
$$

where $z_{1}=\frac{x_{1}-\mu_{\eta}}{\sigma_{\eta}}=\frac{\eta-24.92}{2}, z_{2}=\frac{c-55}{5}, z_{3}=\frac{\varphi-26}{2}$.

The encoded variables in Eq. (8) should be translated into 'natural' uncertain variables; a translated equation can be written as:

$$
\begin{aligned}
\hat{y}_{\text {up-soed }}= & -10.215+0.0006 \eta^{2}+0.00004 c^{2} \\
& +\eta(0.192+0.0002 c-0.0094 \varphi) \\
& +c(0.0858-0.00375 \varphi) \\
& +(0.4377+0.0005 \varphi) \varphi
\end{aligned}
$$

The performance function with respect to a limiting $F S\left(F S_{\text {min }}=1\right)$ is:

$G^{\prime}(x)_{\text {up - soed }}=\hat{y}_{\text {up - soed }}-F S_{\text {min }}=\hat{y}_{\text {up - soed }}-1$

In order to make a comparison, the regression equation is also determined by the conventional RSM, in which the design matrix is based on central composite designs. FS is listed in Table 6. It can be noticed that the products by $\mathrm{z}_{11}{ }^{\prime}$ and $\mathrm{z}_{22}$ ' are not equal to 0 , i.e., the design matrix by a second-order forms does not satisfy the orthogonality in the conventional RSM.
The regression equation by the conventional RSM is:

$$
\begin{aligned}
\hat{y}_{\text {up - RSM }}= & 1.115-0.0217 z_{1}-0.0425 z_{2}+0.0530 z_{3} \\
& +0.002 z_{1} z_{2}-0.0375 z_{1} z_{3}-0.0375 z_{2} z_{3} \\
& +0.0018 z_{11}^{\prime}+0.0006 z_{22}^{\prime}+0.0013 z_{33}^{\prime}
\end{aligned}
$$

A translated equation can be written as:

$$
\begin{aligned}
\hat{y}_{\text {up - RSM }}= & -9.448+0.00045 \eta^{2}+0.000026 c^{2} \\
& +\eta(0.1995+0.0002 c-0.0094 \varphi) \\
& +c(0.08112-0.00375 \varphi) \\
& +(0.4494+0.0003 \varphi) \varphi
\end{aligned}
$$

Similarly, the performance function with respect to a limiting $F S\left(F S_{\min }=1\right)$ is:

$G^{\prime}(x)_{\text {up - RSM }}=\hat{y}_{\text {up - RSM }}-1$

For comparison, the errors between $F S$ obtained by 3D rigorous limit equilibrium method and the proposed method are plotted in Fig. 5, and the errors between $F S$ obtained by $3 \mathrm{D}$ rigorous limit equilibrium method and the conventional RSM are plotted in Fig. 6. It can be found that the standard deviation of errors in Fig. 5 is less than that in Fig. 6. Meanwhile, the mean value of errors for the proposed method is equal to 0 . Such a phenomenon appears because the variance of the error is minimized in the proposed method.

For SOED-based RSM, the reliability index $\beta_{\mathrm{RI}}$ and the probability of failure of the upper sliding mass can be obtained by Eqs. (7) and (10) with the help of MATLAB 
Table 6 Design matrix in the conventional RSM with three variables $(m=3)$

\begin{tabular}{lcrrrrrrrrr}
\hline $\begin{array}{l}\text { Experimental } \\
\text { order }\end{array}$ & $z_{1}$ & $z_{2}$ & $z_{3}$ & $z_{1} z_{2}$ & $z_{1} z_{3}$ & $z_{2} z_{3}$ & $z_{11}{ }^{\prime}$ & $z_{22}{ }^{\prime}$ & $z_{33}{ }^{\prime}$ & \multicolumn{2}{l}{$\begin{array}{l}\text { Response } \\
F S\end{array}$} \\
\hline 1 & 1 & 1 & 1 & 1 & 1 & 1 & 0.09 & 0.09 & 0.09 & 0.996 \\
2 & 1 & 1 & -1 & 1 & -1 & -1 & 0.09 & 0.09 & 0.09 & 1.146 \\
3 & 1 & -1 & 1 & -1 & 1 & -1 & 0.09 & 0.09 & 0.09 & 1.191 \\
4 & 1 & -1 & -1 & -1 & -1 & 1 & 0.09 & 0.09 & 0.09 & 1.041 \\
5 & -1 & 1 & 1 & -1 & -1 & 1 & 0.09 & 0.09 & 0.09 & 1.185 \\
6 & -1 & 1 & -1 & -1 & 1 & -1 & 0.09 & 0.09 & 0.09 & 1.035 \\
7 & -1 & -1 & 1 & 1 & -1 & -1 & 0.09 & 0.09 & 0.09 & 1.238 \\
8 & -1 & -1 & -1 & 1 & 1 & 1 & 0.09 & 0.09 & 0.09 & 1.088 \\
9 & 1.682 & 0 & 0 & 0 & 0 & 0 & 1.918 & -0.91 & -0.91 & 1.08 \\
10 & -1.682 & 0 & 0 & 0 & 0 & 0 & 1.918 & -0.91 & -0.91 & 1.154 \\
11 & 0 & 1.682 & 0 & 0 & 0 & 0 & -0.91 & 1.918 & -0.91 & 1.153 \\
12 & 0 & -1.682 & 0 & 0 & 0 & 0 & -0.91 & 1.918 & -0.91 & 1.071 \\
13 & 0 & 0 & 1.682 & 0 & 0 & 0 & -0.91 & -0.91 & 1.918 & 1.242 \\
14 & 0 & 0 & -1.682 & 0 & 0 & 0 & -0.91 & -0.91 & 1.918 & 0.99 \\
15 & 0 & 0 & 0 & 0 & 0 & 0 & -0.91 & -0.91 & -0.91 & 1.113 \\
\hline
\end{tabular}

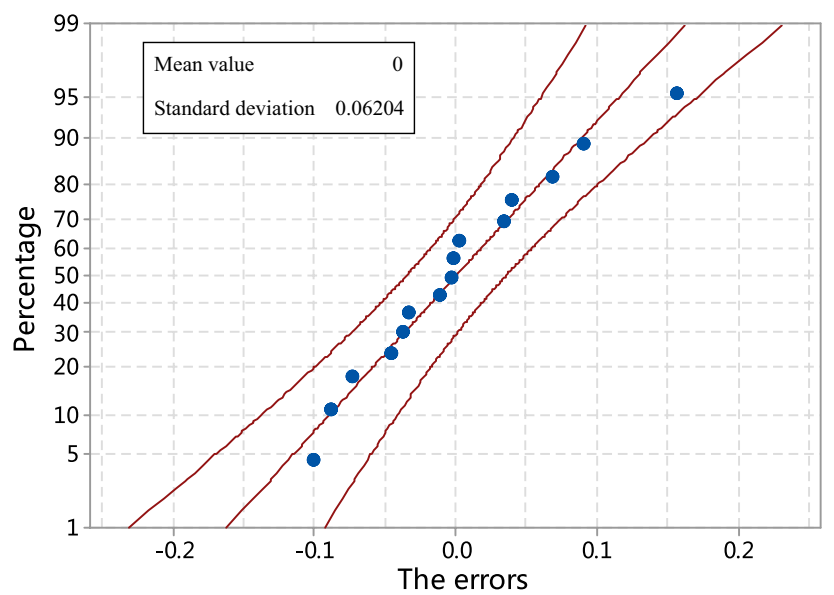

Fig. 5 Errors between FS obtained by 3D rigorous limit equilibrium method and SOED-based RSM

optimization toolbox. Similarly, for the conventional $\mathrm{RSM}$, the reliability index $\beta_{\mathrm{RI}}$ and the probability of failure of the upper sliding mass can be determined by Eqs. (10) and (13). The results are listed in Table 7. It can be found from Table 7 that the probability of failure of the upper sliding mass by the proposed method is in good agreement with that by Monte Carlo simulations (MC), which is larger than that by the conventional RSM. The main reason of such a phenomenon is that the highorder terms in the conventional RSM do not satisfy the orthogonality. That is to say, the second-order response surface for SOED-based RSM is more rigorous than that for the conventional RSM because the orthogonality of variables in high-order design matrix is ignored in the conventional RSM.

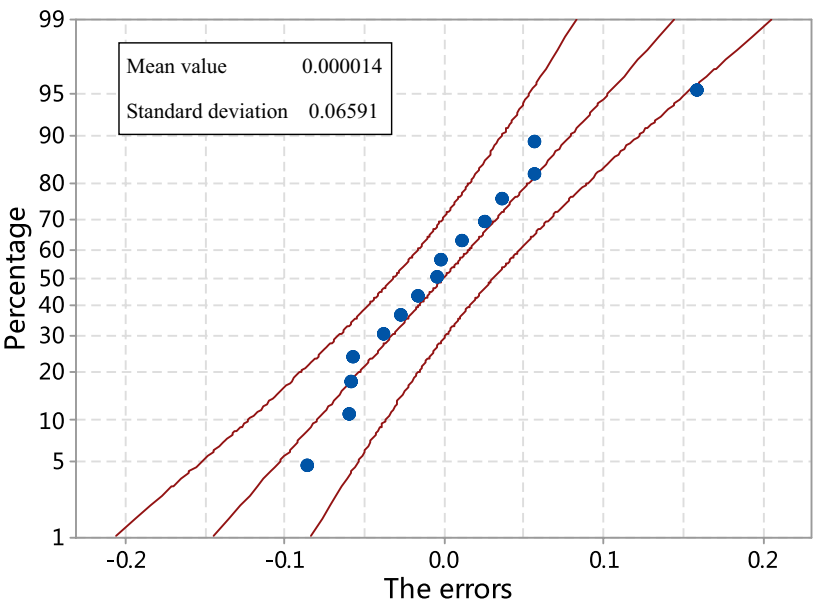

Fig. 6 Errors between FS obtained by 3D rigorous limit equilibrium method and the conventional RSM

\section{The lower sliding mass}

Similarly, the response values of $F S$ of the lower sliding mass are calculated using 3D rigorous limit equilibrium method, and uncertain variables are also transformed from Table 5 . The regression equation by the proposed method can be obtained as follows:

$$
\begin{aligned}
\hat{y}_{\text {low } S O E D}= & 1.6550-0.0681 z_{1}-0.0327 z_{2}+0.0737 z_{3} \\
& +0.0056 z_{1} z_{2}+0.0055 z_{1}^{2}+0.0012 z_{3}^{2}
\end{aligned}
$$

The translated equation for the lower sliding mass can be written as:

$$
\begin{aligned}
\hat{y}_{\text {up }-\operatorname{RSM}}= & 30848+\eta(-0.1055+0.0014 \eta+0.00056 c) \\
& -0.205 c+(0.0219+0.0003 \varphi) \varphi
\end{aligned}
$$


FS obtained by 3D rigorous limit equilibrium method is compared with those obtained by SOED-based RSM, as shown in Figs. 7 and 8. It can be found from Figs. 7 and 8 that FS obtained by the proposed approach is in good agreement with that obtained by $3 \mathrm{D}$ rigorous limit equilibrium method. In order to prove the accuracy of SOED-based RSM, a well-accepted simulation method, Monte Carlo simulations, is employed herein.
The reliability index $\beta_{\mathrm{RI}}$ obtained by SOED-based RSM with the help of MATLAB optimization toolbox is 2.62 , and the probability of failure of the lower sliding mass is $4.4 \%$. The probability of failure obtained by Monte Carlo simulations with $10^{12}$ sampling points is $4.2 \%$. The probability of failure obtained by SOED-based RSM is in good agreement with that obtained by Monte Carlo simulations. It can be found from Table 3 that the lower sliding mass is between
Table 7 Comparison of $\beta_{\mathrm{RI}}$ and the probability of failure $P_{f}$ using the different methods

Fig. 7 FS obtained by 3D rigorous limit equilibrium method and SOED-based RSM

Fig. 8 Errors between FS obtained by 3D rigorous limit equilibrium method and the proposed method

\begin{tabular}{llll}
\hline Methods & $\begin{array}{l}\text { Reliability index } \\
\beta_{\mathrm{RI}}\end{array}$ & $\begin{array}{l}\text { The probability of } \\
\text { failure }(\%)\end{array}$ & Failure points $(\eta, \mathrm{c}, \varphi)$ \\
\hline The proposed method & 1.42 & 7.71 & $(24.842,45.000,24.413)$ \\
The conventional RSM & 1.52 & 6.73 & $(27.209,45.002,22.795)$ \\
Monte Carlo simulations & 1.42 & 7.74 & $/$ \\
\hline
\end{tabular}
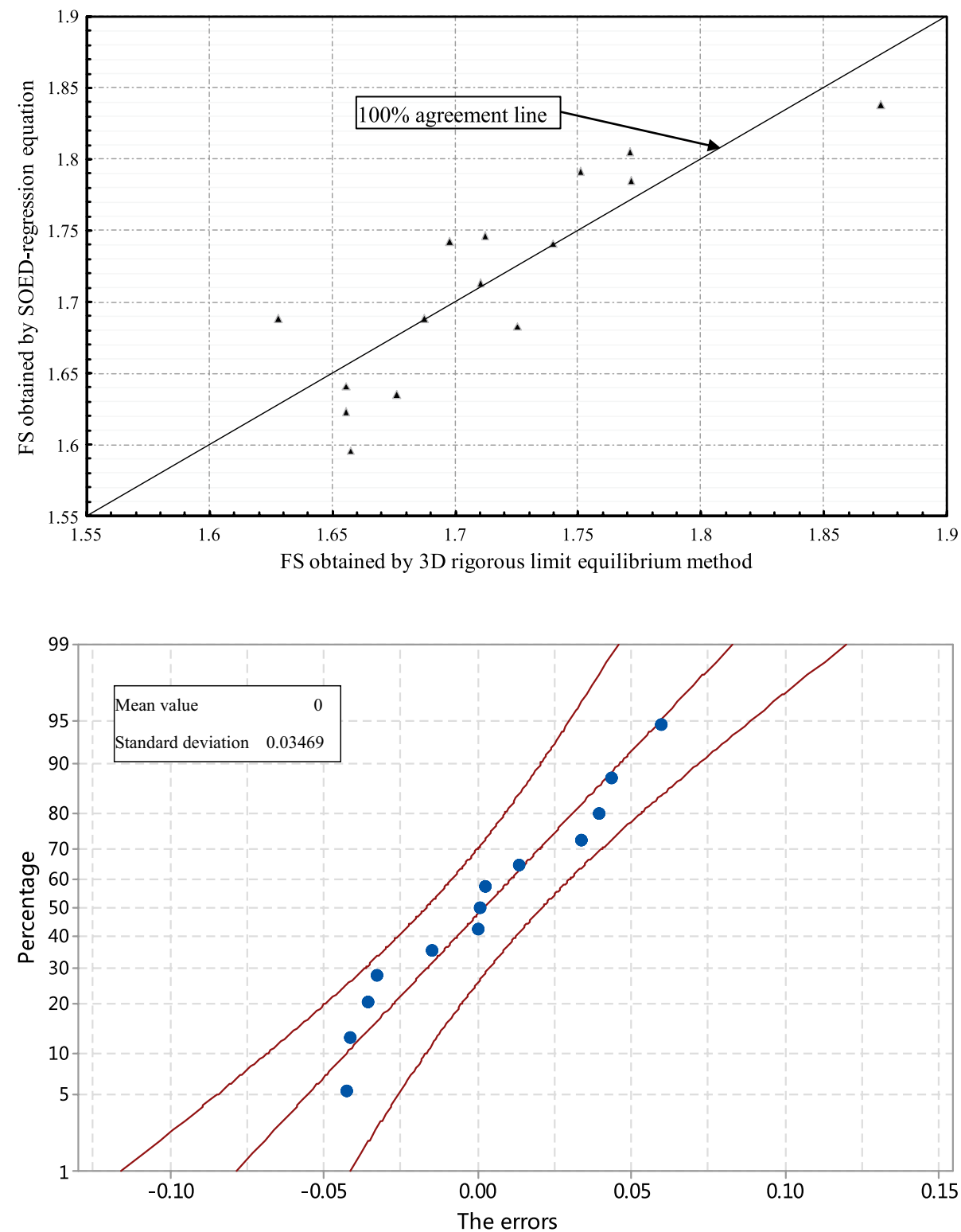
'unsatisfactory' and 'poor,' which means the landslide may fail. That is to say, it should be reinforced as soon as possible. In fact, Xiangjiashan landslide was reinforced.

\section{Summary and conclusions}

Rocks or soils in landslides are highly variable natural materials. In order to deal with uncertainties, reliability analysis approach, which is a reasonable and rigorous way, is applied in landslides or the other engineering. A SOED-based RSM is proposed to study the stability of landslides. Firstly, a second-order design matrix is built by changing the length of star points. Secondly, the unit weight, cohesion and friction angle of geomaterials are considered as random variables, and their values are transformed from the encoded variables in the proposed method. Thirdly, the response value, the safety factor, is calculated by $3 \mathrm{D}$ rigorous limit equilibrium method. Finally, an example is taken to verify the validity of the proposed approach. Compared with the conventional RSM, it can be found that the results obtained by the proposed method are more accurate. Therefore, the proposed method provides a new idea to analyze the stability of landslides.

Acknowledgements The work was supported by the National Natural Science Foundation of China (Nos. 51325903, 51679017) and the Natural Science Foundation Project of CQ CSTC (Nos. cstc2015jcyjys0002, cstc2015jcyjys0009 and cstc2016jcyjys0005).

Open Access This article is distributed under the terms of the Creative Commons Attribution 4.0 International License (http://creativecommons.org/licenses/by/4.0/), which permits unrestricted use, distribution, and reproduction in any medium, provided you give appropriate credit to the original author(s) and the source, provide a link to the Creative Commons license, and indicate if changes were made.

\section{Appendix (Zhou and Cheng 2014) Determination of $F S$ of three-dimensional landslides using 3D rigorous limit equilibrium method}

As shown in Fig. 9, the sliding body is divided into $m$ columns in the $x$-direction and $n$ columns in the $y$-direction. Each column is labeled using indices $i$ and $j$, which, respectively, represent the number of rows and columns. $W^{i, j}$ denotes the weight of the column. $N^{i, j}$ and $S^{i, j}$ are, respectively, the normal force and shear force at the slip surface. The inter-column force between the column $(i, j)$ and the column $(i, j-1)$ is denoted as $Q^{i, j}$. Meanwhile, the intercolumn force between column $(i, j)$ and column $(i-1, j)$ is $G^{i, j}$. The inclinations of inter-column forces of $Q^{i, j}$ are $\pm \alpha$, and the inclinations of inter-column forces of $G^{i, j}$ are $\pm \beta$.

The coordinate system o-xyz is established in Fig. 9, and the entire sliding body is located into the first quadrant. The

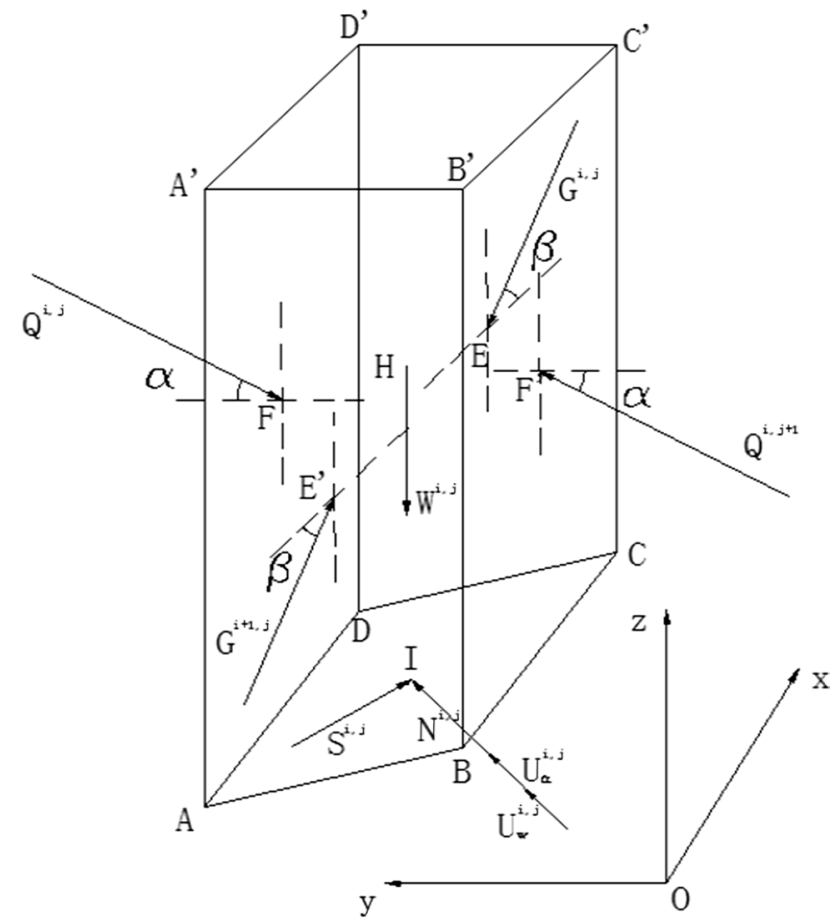

Fig. 9 Geometric elements of the slopes

ground surface and the slip surface are described by equations $\mathrm{z}_{1}=g(x, y)$ and $\mathrm{z}_{2}=f(x, y)$, respectively. The direction cosines of normal forces over the slip surface are denoted as $\left(n_{x}^{i, j}, n_{y}^{i, j}, n_{z}^{i, j}\right)$, and the direction cosines of shear forces over the slip surface are denoted as $\left(l_{x}^{i, j}, l_{y}^{i, j}, l_{z}^{i, j}\right)$.

The direction cosines of normal forces over the slip surface can be described as

$\left(n_{x}^{i, j}, n_{y}^{i, j}, n_{z}^{i, j}\right)=\left(-\frac{1}{\Delta} \frac{\partial f}{\partial x},-\frac{1}{\Delta} \frac{\partial f}{\partial y}, \frac{1}{\Delta}\right)$

where

$\Delta=\sqrt{1+\left(\frac{\partial f}{\partial x}\right)^{2}+\left(\frac{\partial f}{\partial y}\right)^{2}}$

Since $x$-axis is parallel to the moving direction of the sliding block, we have

$\left(l_{x}^{i, j}, l_{y}^{i, j}, l_{z}^{i, j}\right)=\frac{1}{\Delta^{\prime}}\left(1,0, \frac{\partial f}{\partial x}\right)$

where

$\Delta^{\prime}=\sqrt{1+\left(\frac{\partial f}{\partial x}\right)^{2}}$

The weight of the column can be denoted by 
$W^{i, j}=\eta A^{i, j}[g(x, y)-f(x, y)]$

where $\eta$ is the unit weight of geomaterials, and $A^{i, j}$ is the cross-sectional area of the column.

The equation of force equilibrium along the $x$-axis, $y$-axis and $z$-axis is, respectively

$\left(N^{i, j}+U_{\mathrm{w}}^{i, j}+U_{\mathrm{a}}^{i, j}\right) n_{x}+S^{i, j} l_{x}+G^{i+1, j} \cos \beta-G^{i, j} \cos \beta=0$

$\left(N^{i, j}+U_{\mathrm{w}}^{i, j}+U_{\mathrm{a}}^{i, j}\right) n_{y}+S^{i, j} l_{y}+Q^{i, j+1} \cos \alpha-Q^{i, j} \cos \alpha=0$

$\left(N^{i, j}+U_{\mathrm{w}}^{i, j}+U_{\mathrm{a}}^{i, j}\right) n_{z}+S^{i, j} l_{z}+G^{i+1, j} \sin \beta$

$-G^{i, j} \sin \beta+Q^{i, j+1} \sin \alpha-Q^{i, j} \sin \alpha-W^{i, j}=0$

Assuming that there are no supporting structures in the sliding body. Hence, the boundary conditions of inter-column force can be described as $G_{1 \leq j \leq n+1}^{0, j}=0, \quad G_{1 \leq j \leq n+1}^{m+1, j}=0$, $Q_{1 \leq i \leq m+1}^{i, 0}=0, Q_{1 \leq i \leq m+1}^{i, n+1}=0$. Because of the relationship of action and reaction among other inter-column forces, the moments of inter-column forces around the axes are equal to 0 . Moment around the $x$-axis, $y$-axis and $z$-axis is, respectively where $x^{\Delta G^{i j} / \Delta Q^{i, j}}$ is $x$ coordinate of the forces point of $\Delta G^{i, j}$ or $\Delta Q^{i, j}\left(\Delta G^{i, j}\right.$ is the different values of inter-column force acting on the plane $\mathrm{ABB}^{\prime} \mathrm{A}^{\prime}$ and the plane $\mathrm{CDD}^{\prime} \mathrm{C}^{\prime}$, $\Delta G^{i, j}=G^{i+1, j}-G^{i, j} ; \Delta Q^{i, j}$ is the different values of intercolumn force acting on the plane $\mathrm{BCC}^{\prime} \mathrm{B}^{\prime}$ and the plane $\mathrm{ADD}^{\prime} \mathrm{A}^{\prime}, \Delta Q^{i, j}=Q^{i, j+1}-Q^{i, j}$ ), which is equal to the $x$ coordinate of point $\mathrm{H} ; y^{\Delta G^{i, j}} / \Delta Q^{i, j}$ is the y coordinate of the force point of $\Delta G^{i, j}$ or $\Delta Q^{i, j}$, which is equal to the y coordinate of point $\mathrm{H} ; z^{\Delta G^{i, j} / \Delta Q^{i, j}}$ is the $\mathrm{z}$ coordinate of the force point of $\Delta G^{i, j}$ or $\Delta Q^{i, j}$, which is equal to the $\mathrm{z}$ coordinate of point $\mathrm{H}$; $x_{i, j}^{H}$ is the $\mathrm{x}$ coordinate of point $\mathrm{H}$; and $y_{i, j}^{H}$ is the y coordinate of point $\mathrm{H}$.

Following Coulombs failure criterion, the safety factor of a slope against sliding is given by the following formula

$S^{i, j}=\frac{c A^{i, j}+\left(N^{i, j}-U^{i, j}\right) \tan \varphi}{F S}$

where $c$ is cohesion of the failure discontinuity, $\varphi$ is the effective internal friction angle and the factor of safety is $F S$.

Substituting Eq. (27) into Eqs. (21)-(23) and eliminating $G^{i, j}$ and $Q^{i, j}$, the following expressions can be obtained,

$$
\sum_{i=1}^{m} \sum_{j=1}^{n}\left\{-\left[\left(N^{i, j}+U_{w}^{i, j}+U_{a}^{i, j}\right) n_{i, j}^{y}+S^{i, j} l_{i, j}^{y}\right] z^{\Delta G^{i, j} / \Delta Q^{i, j}}+\left[\left(N^{i, j}+U_{w}^{i, j}+U_{a}^{i, j}\right) n_{i, j}^{z}+S^{i, j} l_{i, j}^{z}\right] y^{\Delta G^{i, j} / \Delta Q^{i, j}}-W^{i, j} y_{i, j}^{H}\right\}=0
$$

$\sum_{i=1}^{m} \sum_{j=1}^{n}\left\{\left[\left(N^{i, j}+U_{w}^{i, j}+U_{a}^{i, j}\right) n_{i, j}^{x}+S^{i, j} l_{i, j}^{x}\right] z^{\Delta G^{i, j} / \Delta Q^{i, j}}-\left[\left(N^{i, j}+U_{w}^{i, j}+U_{a}^{i, j}\right) n_{i, j}^{z}+S^{i, j} l_{i, j}^{z}\right] x^{\Delta G^{i, j} / \Delta Q^{i, j}}+W^{i, j} x_{i, j}^{H}\right\}=0$

$\sum_{i=1}^{m} \sum_{j=1}^{n}\left\{-\left[\left(N^{i, j}+U_{w}^{i, j}+U_{a}^{i, j}\right) n_{i, j}^{x}+S^{i, j} l_{i, j}^{x}\right] y^{\Delta G^{i, j} / \Delta Q^{i, j}}+\left[\left(N^{i, j}+U_{w}^{i, j}+U_{a}^{i, j}\right) n_{i, j}^{y}+S^{i, j} l_{i, j}^{y}\right] x^{\Delta G^{i, j} / \Delta Q^{i, j}}\right\}=0$ 


$$
\begin{aligned}
N^{i, j}= & \frac{\cos \alpha \sin \beta\left(c A^{i, j} l_{x}^{i, j}+F S n_{x}^{i, j} U^{i, j}-l_{x}^{i, j} U^{i, j} \tan \varphi\right)+\cos \beta \sin \alpha\left(c A^{i, j} l_{y}^{i, j}+F S n_{y}^{i, j} U^{i, j}-l_{y}^{i, j} U^{i, j} \tan \varphi\right)}{-\cos \alpha \sin \beta\left(F S n_{x}^{i, j}+l_{x}^{i, j} \tan \varphi\right)-\left(\cos \beta \sin \alpha\left(F S n_{y}^{i, j}+l_{y}^{i, j} \tan \varphi\right)\right)+\left(\cos \alpha \sin \beta\left(F S n_{z}^{i, j}+l_{z}^{i, j} \tan \varphi\right)\right)} \\
& +\frac{\cos \alpha \cos \beta\left(-c A^{i, j} l_{z}^{i, j}-F S n_{z}^{i, j} U^{i, j}+l_{z}^{i, j} U^{i, j} \tan \varphi\right)+\cos \beta \cos \alpha F S W^{i, j}}{-\cos \alpha \sin \beta\left(F S n_{x}^{i, j}+l_{x}^{i, j} \tan \varphi\right)-\left(\cos \beta \sin \alpha\left(F S n_{y}^{i, j}+l_{y}^{i, j} \tan \varphi\right)\right)+\left(\cos \alpha \sin \beta\left(F S n_{z}^{i, j}+l_{z}^{i, j} \tan \varphi\right)\right)}
\end{aligned}
$$

$$
\begin{aligned}
S^{i, j}= & \frac{n_{x}^{i, j} \cos \alpha \sin \beta\left(c A^{i, j} l_{x}^{i, j}-2 U^{i, j} \tan \varphi\right)+n_{y}^{i, j} \sin \alpha \cos \beta\left(c A^{i, j}-2 U^{i, j} \tan \varphi\right)}{\cos \alpha \sin \beta\left(F S n_{x}^{i, j}+l_{x}^{i, j} \tan \varphi\right)+\cos \beta \sin \alpha\left(F S n_{y}^{i, j}+l_{y}^{i, j} \tan \varphi\right)-\cos \alpha \sin \beta\left(F S n_{z}^{i, j}+l_{z}^{i, j} \tan \varphi\right)} \\
& -\frac{n_{z}^{i, j} \cos \alpha \cos \beta\left(c A^{i, j} l_{z}^{i, j}-2 U^{i, j} \tan \varphi\right)+\cos \beta \cos \alpha W^{i, j} \tan \varphi}{\cos \alpha \sin \beta\left(F S n_{x}^{i, j}+l_{x}^{i, j} \tan \varphi\right)+\cos \beta \sin \alpha\left(F S n_{y}^{i, j}+l_{y}^{i, j} \tan \varphi\right)-\cos \alpha \sin \beta\left(F S n_{z}^{i, j}+l_{z}^{i, j} \tan \varphi\right)}
\end{aligned}
$$

Substituting $N^{i, j}, S^{i, j}$ of Eqs. (28) and (29) into Eqs. (24)-(26), the following expressions can be written as

$$
\left\{\begin{array}{l}
F_{1}(\alpha, \beta, F s)=\sum M_{x}=0 \\
F_{2}(\alpha, \beta, F s)=\sum M_{y}=0 \\
F_{3}(\alpha, \beta, F s)=\sum M_{z}=0
\end{array}\right.
$$

The set of nonlinear Eq. (30) can be solved using trustregion-reflective iterative algorithm. The initial value is set as $\alpha=0, \beta=0$ and $F S=1$. The local optimal solutions can then be obtained using 10-20 iterations. The value of $F S$ of three-dimensional slopes can be determined finally.

\section{References}

Box GEP, Draper NR (2006) Introduction to response surface methodology. Response surfaces, mixtures, and ridge analyses, 2nd edn. Wiley, Hoboken

Ching J, Phoon KK (2012) Value of geotechnical site investigation in reliability-based design. Adv Struct Eng 15:1935-1946

Duncan JM, Buchignani AL (1973) Failure of underwater slope in San Francisco bay. J Soil Mech Found Div 9:687-703

Guan XL, Melchers RE (2001) Effect of response surface parameter variation on structural reliability estimates. Struct Saf 23:429-444

Hall M (1959) Orthogonal Latin squares. Proc Natl Acad Sci USA 45:859-862

He K, Wang S, Du W, Wang S (2010) Dynamic features and effects of rainfall on landslides in the three gorges reservoir region, China: using the Xintan landslide and the large Huangya landslide as the examples. Environ Earth Sci 59:1267-1274

Hicks MA, Nuttall JD, Chen J (2014) Influence of heterogeneity on $3 \mathrm{~d}$ slope reliability and failure consequence. Comput Geotech 61:198-208
Ji J, Liao HJ, Low BK (2012) Modeling 2-D spatial variation in slope reliability analysis using interpolated autocorrelations. Comput Geotech 40:135-146

Johari A, Lari AM (2016) System probabilistic model of rock slope stability considering correlated failure modes. Comput Geotech 81:26-38

Johnson DW, Johnson FP (1991) Joining together: group theory and group skills, 4th edn. Allyn and Bacon, Boston

Li D, Zhou C, Lu W, Jiang Q (2009) A system reliability approach for evaluating stability of rock wedges with correlated failure modes. Comput Geotech 36:1298-1307

Li DQ, Chen YF, Lu WB, Zhou CB (2011) Stochastic response surface method for reliability analysis of rock slopes involving correlated non-normal variables. Comput Geotech 38:58-68

Li DQ, Zheng D, Cao ZJ, Tang XS, Phoon KK (2015) Response surface methods for slope reliability analysis: review and comparison. Eng Geol 203:3-14

Low BK (2013) FORM, SORM, and spatial modeling in geotechnical engineering. Struct Saf 49:56-64

Myers H, Raymond H (2012) Response surface methodology: process and product optimization using designed experiments, 2nd edn. Wiley, Hoboken

Phoon KK (2008) Reliability-based design in geotechnical engineering: computations and applications. CRC Press, London

Phoon KK, Kulhawy FH (1999) Characterization of geotechnical variability. Can Geotech J 36:612-624

Pourghasemi HR, Moradi HR, Aghda SMF, Sezer EA, Jirandeh AG, Pradhan B (2014) Assessment of fractal dimension and geometrical characteristics of the landslides identified in north of Tehran, Iran. Environ Earth Sci 71:3617-3626. https://doi.org/10.1007/ s12665-013-2753-9

Tang XS, Li DQ, Rong G, Phoon KK, Zhou CB (2013) Impact of copula selection on geotechnical reliability under incomplete probability information. Comput Geotech 49:264-278

US Army Corps of Engineers (1995) Introduction to probability and reliability methods for use in geotechnical engineering, in ETL $1110-2-547$

Wang Y (2012) Uncertain parameter sensitivity in Monte Carlo simulation by sample reassembling. Comput Geotech 46:39-47

Wang Y, Cao Z, Au SK (2010) Efficient Monte Carlo Simulation of parameter sensitivity in probabilistic slope stability analysis. Comput Geotech 37(7):1015-1022 
Wang N, Shi T, Li Y, Lian Z, Ke P (2016) Quantitative assessment on the influence of rainfall on landslides in Jianshi County of Qing River Basin, China. Environ Earth Sci 75:241. https://doi. org/10.1007/s12665-015-4899-0

Zhang J, Huang HW, Phoon KK (2012) Application of the Krigingbased response surface method to the system reliability of soil slopes. J Geotech Geoenviron 139:651-655
Zhou XP, Cheng H (2014) Stability analysis of three-dimensional seismic landslides using the rigorous limit equilibrium method. Eng Geol 174:87-102

Zhou XP, Cheng H (2015) The long-term stability analysis of 3D creeping slopes using the displacement-based rigorous limit equilibrium method. Eng Geol 195:292-300 